\title{
ABSORPTION AND EMISSION SPECTRA FOR C60 ANIONS
}

\author{
TATSUHISA KATO \\ Institute for Molecular Science, Myodaiji, Okazaki 444, Japan
}

(Received 14 May 1993)

\begin{abstract}
Absorption spectra are detected for $\mathrm{C}_{60}^{-}$and $\mathrm{C}_{60}^{2-}$ produced electrolytically in solution at room temperature. Theoretical analysis of the spectrum of $\mathrm{C}_{60}^{-}$by $\mathrm{CNDO} / \mathrm{S}$ calculations gives an interpretation of the characteristic near-IR bands, the weak visible bands, and the strong bands in the UV region. The emission spectrum of $\mathrm{C}_{60}^{-}$is a mirror image of the near-IR absorption band, and the detection of the emission reconfirms our original assignment of the absorption spectrum. The nature of the spectrum of $\mathrm{C}_{60}^{2-}$ is characterized by a similar orbital picture to that of $\mathrm{C}_{60}^{-}$. Further laser experiments of significance are proposed.
\end{abstract}

KEY WORDS: $\mathrm{C}_{60}$ negative ion, near IR absorption, CNDO calculation, Jahn-Teller distortion

\section{INTRODUCTION}

Since our report of the electronic absorption spectra of the radical cation $\left(\mathrm{C}_{60}^{+}\right)$and anion $\left(\mathrm{C}_{60}^{-}\right)$in $\gamma$-irradiated glassy polyatomic matrices at $77 \mathrm{~K}^{1}$, many investigations of these radical ions have been performed. Similar spectra have been obtained not only in Ar matrix ${ }^{2}$ but also in the electrolyzed solutions ${ }^{3,45,6}$, but some contradictory interpretations were reported as to the nature of the near-IR absorption band of $\mathrm{C}_{60}^{-}$. In order to settle this contradiction, a consistent interpretation for the electronic absorption and newly detected emission spectra of $\mathrm{C}_{60}^{-7}$ are proposed here in connection with the assignment of the absorption band of $\mathrm{C}_{60}^{2-}$.

\section{EXPERIMENTAL}

$\mathrm{C}_{60}$ was prepared by a method similar to that described by Krätschmer et al. ${ }^{8} . \mathrm{C}_{60}^{-}$ and $\mathrm{C}_{60}^{2-}$ were produced by controlled-potential electrolysis using a flow cell equipped with a reference electrode. The solvent and the electrolyte used for electrolysis are methylenechloride and tetra-n-butyl ammonium perchlorate purified by standard procedures. The solution of methylenechloride containing about $2.5 \times 10^{-4} \mathrm{~mol} / \mathrm{l}$ of $\mathrm{C}_{60}$ and about $0.1 \mathrm{~mol} / \mathrm{l}$ of the electrolyte was deoxygenated by argon gas bubbling. The 
quantitative-electrolysis method used here can achieve $100 \%$ efficiency for the electrochemical reduction; the concentration of mono- and di- anions can be controlled quantitatively by adjusting the electrode potential and the flow rate of the solution. Potentials of $-0.8 \mathrm{~V}$ and $-1.6 \mathrm{~V}$ vs. $\mathrm{I}_{2} / \mathrm{I}^{-}$(acetonitrile) were used to reduce $\mathrm{C}_{60}$ to the mono- and di- anion. In this way the extinction coefficient of $\mathrm{C}_{60}^{-}$at $1,073 \mathrm{~nm}$ was determined as $1.55 \times 10^{4} 1 \mathrm{~mol}^{-1} \mathrm{~cm}^{-1}$ and that of $\mathrm{C}_{60}^{2-}$ at $950 \mathrm{~nm}$ as $2.09 \times 10^{4} \mathrm{lmol}^{-1} \mathrm{~cm}^{-1}$. The absorptions were recorded on a Hitachi U-3400 spectrometer and the emission spectrum by a Bomem DA 3.36 FT spectrometer in combination with a Nd:YAG laser.

\section{RESULTS AND DISCUSSION}

The spectra for $\mathrm{C}_{60}^{-}$and $\mathrm{C}_{60}^{2-}$ are shown in Figure 1. Both spectra reveal specific near-IR bands starting at $1073 \mathrm{~nm}$ for $\mathrm{C}_{60}^{-}$and $950 \mathrm{~nm}$ for $\mathrm{C}_{60}^{2-}$, weak visible bands around $600 \mathrm{~nm}$, and very intense UV bands. Although the detailed band shape in the UV region was not obtained because of overlapping absorption due to the neutral $\mathrm{C}_{60}$ and the electrolyte, an increasing absorbance was measured.
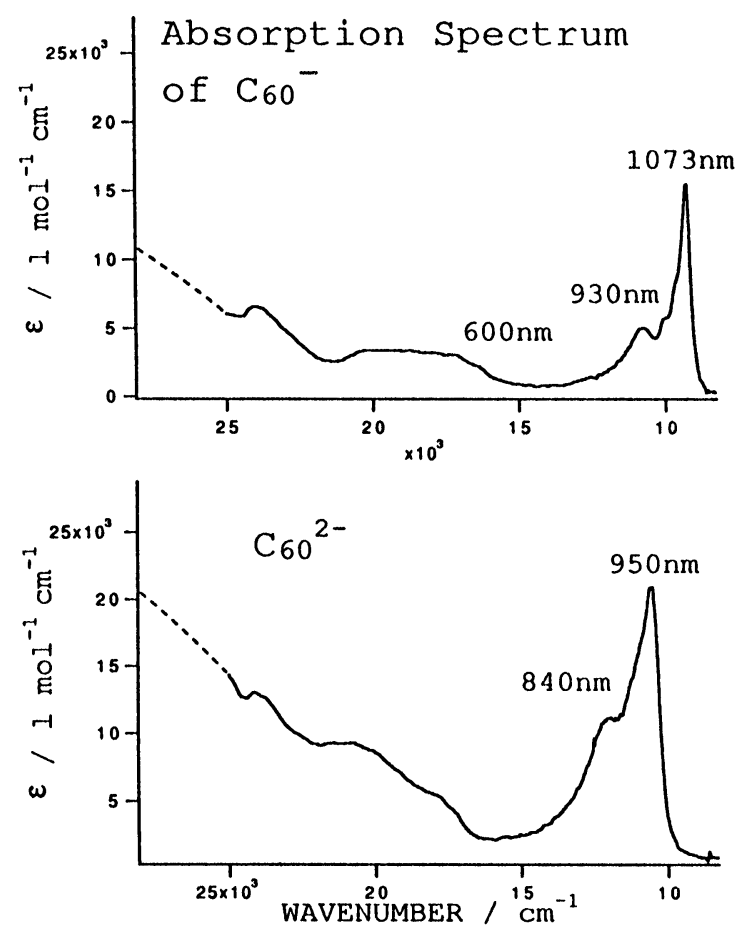

Figure 1 Absorption spectra for $\mathrm{C}_{60}^{-}$and $\mathrm{C}_{60}^{2-}$ produced electrolytically in solution at room temperature. 
Figure 2 shows the emission spectrum of $\mathrm{C}_{60}^{-}$obtained with a ND:YAG laser excitation at $1,064 \mathrm{~nm}$ together with the absorption spectrum. The emission spectrum is a mirror image of the near-IR absorption band, and the emission and absorption spectra both show vibrational progressions with fundamentals of 1,310 and $390 \mathrm{~cm}^{-1}$, and 1,410 and $370 \mathrm{~cm}^{-1}$ respectively. The mirror image pattern of emission and absorption spectra supports our assignment ${ }^{1}$ of the near-IR bands at $1,073 \mathrm{~nm}$ and $930 \mathrm{~nm}$ to vibronic structure, not to two different electronic states as suggested by Negri et al. ${ }^{9}$ or Lawson et al. ${ }^{6}$ The near-IR absorption band can be characterized as the first optically allowed transition on the basis of results obtained by our $\mathrm{CNDO} / \mathrm{S}^{1}$ calculation and a SCF-X $\alpha$-SW calculation by Heath et al. ${ }^{5}$ Negri et al., ${ }^{9}$ however, proposed a different assignment of the band, namely to a second optically allowed transition, from the results of PPP calculation. The detection of the emission band favors our interpretation. The near-IR band indeed corresponds to the first optically allowed transition unless $\mathrm{C}_{60}^{-}$belongs to the exceptional case of a breakdown in Kasha's law.

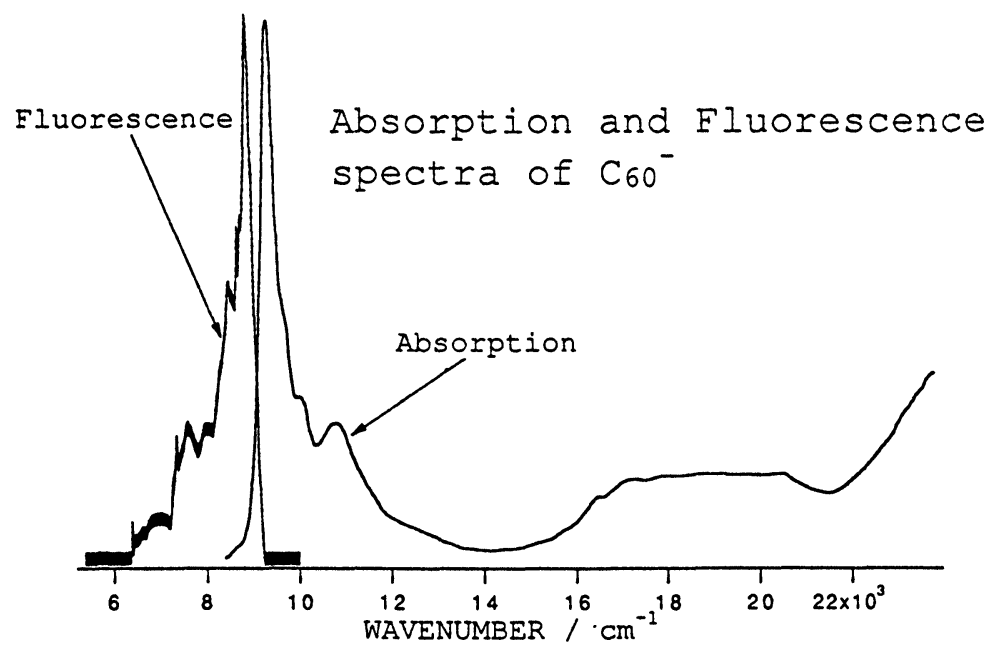

Figure 2 Absorption and emission spectra of $\mathrm{C}_{60}^{-}$produced by electrolysis and measured at room temperature.

This experimental support for our assignment encourages us to interpret the absorption bands of $\mathrm{C}_{60}^{-}$and $\mathrm{C}_{60}^{2-}$ consistently in terms of the results of a revised $\mathrm{CNDO} / \mathrm{S}$ calculation. Figure 3 shows the absorption spectrum of $\mathrm{C}_{60}^{-}$together with a stick diagram which exhibits the oscillator strength at each transition energy obtained by the CNDO/S calculation. The CNDO/S calculation was done by using the Jahn-Teller distorted molecular geometry which Koga et al. ${ }^{10}$ optimized at ab-initio level of theory. They showed that small Jahn-Teller distortions took place to give the $D_{2 h}, D_{3 d}$, and $D_{5 d}$ symmetry structures. The results of the CNDO/S calculation for the $D_{2 h}$ symmetry are shown in Figure 3. The near-IR band is well associated 


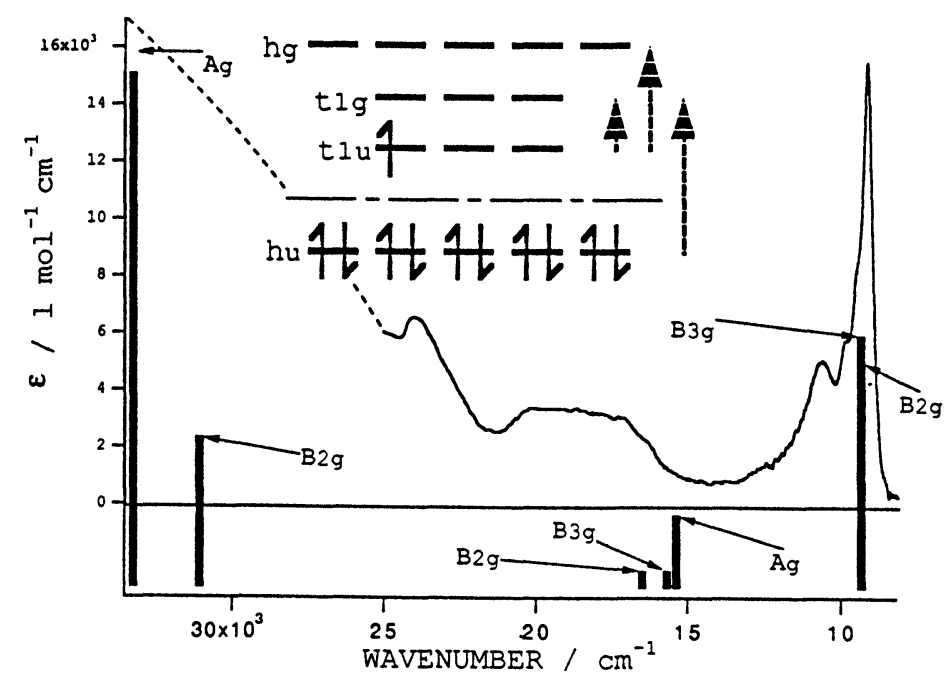

Figure 3 Absorption spectrum for $\mathrm{C}_{60}^{-}$with a stick diagram exhibiting the relative oscillator strengths obtained by CNDO/S calculation for an optimized geometry of $\mathrm{C}_{60}^{-}$in the $\mathrm{D}_{2 \mathrm{~h}}$ symmetry. The inset shows the schematic representation of the three patterns of allowed transitions of $\mathrm{C}_{60}^{-}$in terms of molecular orbitals.

with the $\mathrm{B}_{1 \mathrm{u}} \rightarrow \mathrm{B}_{2 \mathrm{~g}}$ and $\mathrm{B}_{1 \mathrm{u}} \rightarrow \mathrm{B}_{3 \mathrm{~g}}$ transitions, the weak visible band with the $\mathrm{B}_{1 \mathrm{u}}$ $\rightarrow A_{g}, B_{1 u} \rightarrow B_{3 g}$, and $B_{1 u} \rightarrow B_{2 g}$ transitions, and the intense UV band with the $B_{1 u}$ $\rightarrow \mathrm{B}_{2 \mathrm{~g}}$ and $\mathrm{B}_{1 \mathrm{u}} \rightarrow \mathrm{A}_{\mathrm{g}}$ transitions. Considering the small amplitude of the Jahn-Teller distortion as shown in the theoretical calculation by Koga et al., and the correlation of the state between the $D_{2 h}$ and hypothetical $I_{h}$ symmetries, the specific features of the absorption bands of $\mathrm{C}_{60}^{-}$are classified into three transitions as shown in the inset of Figure 3. The near-IR band is approximately interpreted in the orbital picture as an electron jumping from a $t_{1 u}$ to a $t_{1 g}$ orbital $\left(t_{1 u} \rightarrow t_{1 g}\right.$ jump), the visible band as a $t_{1 u} \rightarrow h_{g}$ jump, and the intense band in UV region as $a h_{u} \rightarrow t_{1 g}$ jump. Furthermore, the absorption bands of $\mathrm{C}_{60}^{2-}$ would be also characterized by the same orbital picture. An ESR measurement ${ }^{11}$ has indicated that the ground state of $\mathrm{C}_{60}^{2-}$ is a triplet state, thus two electrons having parallel spin are assigned to two of the three $t_{1 u}$ orbitals, and the near-IR, visible, and UV bands for $\mathrm{C}_{60}^{2-}$ correspond to the optically allowed first, second, and third transitions similar in nature to those of $\mathrm{C}_{60}^{-}$as shown in the inset of Figure 3.

The electronic absorption spectra of $\mathrm{C}_{60}^{-}$and $\mathrm{C}_{60}^{2-}$ measured at room temperature in solution are well interpreted by the approximate orbital picture without considering any Jahn-Teller distortion. On the other hand we reported that the effect of the Jahn-Teller distortion was reflected in the value of the $\mathrm{g}$ factor $^{4}$ and the spectral line shape ${ }^{7}$ of the ESR measurement for $\mathrm{C}_{60}^{-}$. But neither the magnitude nor the direction of the Jahn-Teller distortion could be obtained from the ESR spectra of $\mathrm{C}_{60}^{-}$. In this regard, it is worth pointing out that two further experiments using a laser are possible and significant for investigating the detailed electronic and 
molecular structure of Jahn-Teller distorted $\mathrm{C}_{60^{-}}$. One is an electronic resonance Raman measurement for $\mathrm{C}_{60}^{-}$in solution. Excited states which are lifted from the $\mathrm{T}_{\mathrm{lu}}$ ground state in $\mathrm{I}_{\mathrm{h}}$ symmetry by the Jahn-Teller distortion lie close in energy with the ground state. For example for the geometry in $D_{2 h}$ symmetry, $B_{1 u}, B_{2 u}$, and $B_{3 u}$ states are lifted from the $T_{1 u}$ state and the amount of the splitting energy was calculated as about $2,100 \mathrm{~cm}^{-1}$ by Koga et al. The small energy difference between $B_{2 u}, B_{3 u}$ and $B_{1 u}$ would be measured as the value of the Raman shift. $A$ further experiment of interest is the resonantly enhanced multiphoton ionization of $\mathrm{C}_{60}^{-}$in the gas phase. The electron affinity of $2.6-2.8 \mathrm{eV}$ for $\mathrm{C}_{60}{ }^{12}$ is much larger than the transition energy of $\mathrm{C}_{60}^{-}$to the first excited state at $1,073 \mathrm{~nm}$. Therefore, the photodetachment via the resonant excitation of the state could be detected, and the energy levels for the (ro)vibronic structure in the excited state of $\mathrm{C}_{60}^{-}$could be obtained.

\section{CONCLUSION}

The detection of the emission spectrum of $\mathrm{C}_{60}^{-}$gives strong support to our original assignment of the first electronic absorption band. The features of the absorption bands for both $\mathrm{C}_{60}^{-}$and $\mathrm{C}_{60}^{2-}$ are consistently interpreted by this assignment.

\section{Acknowledgment}

The author is grateful to Dr. M. Oyama and Mr. T. Kodama for help with the controlled potential electrolysis, and to Professor T. Shida for valuable discussions. I am indebted to Professor K. Morokuma and Dr. N. Koga for providing the molecular coordinates of $\mathrm{C}_{60}^{-}$. Thanks are also due to the Instrument Center, Institute for Molecular Science, for the use of a Hitachi U-3400 spectrometer and Bomem DA 3.36 FT spectrometer. This work was supported in part by the Grants-in-Aid for Scientific Research (Grant Nos. 03NP0301 and 0533108) from Ministry of Education, Science and Culture.

\section{References}

1. T. Kato, T. Kodama, T. Shida, T. Nakagawa, Y. Matsui, S. Suzuki, H. Shiromaru, K. Yamauchi, Y. Achiba. Chem. Phys. Lett., 180, 446-450 (1991).

2. L. Andrews. J. Phys. Chem., 96, 1525-1527 (1992).

3. M. A. Greaney, S. M. Gorun. J. Phys. Chem., 95, 7142-7144 (1991).

4. T. Kato, T. Kodama, M. Oyama, S. Okazaki, T. Shida, T. Nakagawa, Y. Matsui, S. Suzuki, H. Shiromaru, K. Yamauchi, Y. Achiba. Chem. Phys. Lett., 186, 35-39 (1991).

5. G. A. Heath, J. E. McGrady, R. L. Martin. Chem. Commun., 1272-1274 (1992).

6. D. R. Lawson, D. L. Feldheim, C. A. Foss, P. K. Dorhout, C. M. Elliott, C. R. Martin, B. Parkinson. J. Electrochem. Soc., 139, L68-L71 (1992).

7. T. Kato, T. Kodama, T. Shida. Chem. Phys. Lett., 205, 405-409 (1993).

8. W. Krätschmer, L. D. Lamb, K. Fostiropoulos, D. R. Huffman. Nature., 347, 354 (1990).

9. F. Negri, G. Orlandi, F. Zerbetto. J. Am. Chem Soc., 114, 2909-2913 (1992). 
10. N. Koga, K. Morokuma. Chem. Phys. Lett., 196, 191-196 (1992).

11. D. Dubois, M. T. Jones, K. M. Kadish. J. Am. Chem. Soc., 114, 6446-6451 (1992).

12. S. H. Yang, C. L. Pettiette, J. Conceicao, O. Cheshnovsky, R. E. Smalley. Chem. Phys. Lett., 139, 233 (1987). 